\title{
Methods for Wildfire Spread Prediction and Their Integration With Remote Sensing Data
}

\author{
Sergey A. Khvostikov, Sergey A. Bartalev \\ Space Research Institute RAS, Moscow, Russia \\ khvostikov@d902.iki.rssi.ru
}

\begin{abstract}
This short review article presents description of a field of wildfire modelling and use of remote sensing in this field. There are many wildfire models based on various approaches (physical, empirical, mathematical). Development of remote sensing in recent decades provided vast array of data that can be used by wildfire models and can help evaluate their accuracy. Joint use of remote sensing data and wildfire models can help to tune models and increase their accuracy and opens a way to create automatic just-in-time wildfire danger prediction system. Also in last 10 years there was a notable development in methods of remote sensing data assimilation into wildfire models, which leads to better estimation of wildfire state and higher accuracy of forecast.
\end{abstract}

Keywords: wildfires, fire spread modelling, remote sensing, data assimilation

Copyright (C2019 by the paper's authors. Copying permitted for private and academic purposes 


\title{
МЕТОДЫ ПРОГНОЗНОГО МОДЕЛИРОВАНИЯ РАЗВИТИЯ ПРИРОДНЫХ ПОЖАРОВ С ИСПОЛЬЗОВАНИЕМ ДАННЫХ СПУТНИКОВОГО МОНИТОРИНГА
}

\author{
С.А. Хвостиков, С.А. Барталев \\ Институт космических исследований РАН, Москва, Россия \\ khvostikov@d902.iki.rssi.ru
}

\begin{abstract}
Статья посвящена краткому описанию методов прогнозного моделирования динамики развития природных пожаров с использованием информации, получаемой на основе данных спутникового мониторинга. Модели природных пожаров позволяют на основе разных подходов (физико-химических, эмпирических, математических) имитировать развитие очагов горения. Развитие методов дистанционного зондирования Земли в последние несколько десятилетий позволило получать большой объем данных, которые могут применяться при моделировании пожаров и для оценки достоверности модельных прогнозов. Комбинирование информации спутникового мониторинга развития пожаров и соответствующих моделей позволяет проводить более детальную оценку точности и настройку последних, делая возможным создание систем оперативного автоматического прогнозирования развития фронта горения. Кроме этого в последние примерно десять лет наблюдается активное развитие методов ассимиляции данных дистанционного зондирования Земли в модели, позволяющие уточнить текущее состояния пожара и улучшить дальнейший прогноз его развития.
\end{abstract}

Ключевые слова: природные пожары, моделирование распространения огня, дистанционное зондирование Земли, ассимиляция данных

Природный пожар представляет собой сложное природное явление, динамика которого определяется рядом физических и химических процессов (горение, пиролиз, адвекция, излучение) [1][2][3], происходящих в неоднородной среде, и находящихся во взаимно обратной связи с движением воздушных потоков в атмосфере [4][5]. Ежегодно природные пожары проходят миллионы гектар территории России, представляя серьезную угрозу лесным и другим типам наземных экосистем, объектам инфраструктуры, населенным пунктам. Для оценки угрозы природного пожара и минимизации ущерба от него необходимо осуществлять наблюдение его динамики и иметь возможность прогнозировать его развитие. В статье будут рассмотрены основные направления в развитии методов моделирования динамики пожара и подходы по интеграции в модели данных спутникового мониторинга.

Методы моделирования распространения огня позволяет имитировать развитие пожара и прогнозировать его динамику для оценки степени угрозы и решения задач управления силами и средствами тушения. На данный момент существует большое количество моделей развития пожара, различающихся по области применения и по лежащим в их основе принципам. Можно выделить три основных подхода к моделированию распространения огня. Физико-химические модели [1][2][3][6] воспроизводят основные процессы, происходящие в процессе горения, 
пиролиз, горение, перенос энергии с помощью конвекции, излучения и горящих частиц (искр). Имитационные модели [7][8][9][10] опираются на эмпирически определенные зависимости между процессом распространения огня и условиями горения. Математические модели [11] основываются на некоторой математической абстракции (клеточные автоматы, самоорганизованная критичность и др.) для описания развития пожара. Также модели можно разделить по методу воспроизведения распространения пожара на растровые модели, основанные на регулярной сетки и переходе огня между ее клетками, и векторные модели, основанные на имитации движения периметра пожара [12]. В последнее время по мере увеличения вычислительных мощностей получили развитие вероятностные модели развития пожара, которые вычисляют вероятность достижения пожаром его близлежащей окрестности с учетом различных метеорологических сценариев и/или с учетом погрешностей модели и входных данных [13][14]. Программное обеспечение, построенное на основе моделей развития пожаров (преимущественно эмпирических) находит широкое применение в задачах тренировки пожарных, планирования контролируемых выжиганий, постпожарной оценки эффективности тушения, оценки потенциальной опасности пожаров [12][15][16].

Практическое применение моделей развития пожаров требует наличия оперативных данных о положении фронта и условиях горения. Данные спутникового мониторинга позволяют получать такую информацию регулярно, оперативно и на больших территориях. Например, полученные на основе спутниковых данных цифровые карты рельефа, такие как SRTM [17] и ASTER [18] часто используются при моделировании пожаров. Данные дистанционного зондирования Земли (ДЗ3) также могут использоваться для построения карт горючих материалов [19][20], предоставляющих необходимую для моделей параметризацию условий распространения огня для конкретных типов наземных экосистем. Оперативная информация детектирования очагов активного горения, например, по данным инструмента MODIS [21] или NPP-VIIRS [22][23], может также использоваться для определения исходного положения фронта пожара при моделировании его развития [24][25]. Также в последнее время наблюдается интерес к вопросу оперативного детектирования очагов горения на основе геостационарных спутников, таких как Meteosat (SEVIRI), HIMAWARI (AHI).

Спутниковые данные позволяют получать информацию о динамике пожара с разной степенью оперативности и детальности [26]. Эта информация может использоваться для оценки точности моделей [27][28], а также для настройки их параметров [25][29]. Интеграция данных спутникового мониторинга и моделей развития пожаров позволяет строить системы оценки потенциальных повреждений от активных пожаров, решающие задачи поддержки принятия решения о необходимых противопожарных мерах [25][30][31][32].

В последнее время активно развиваются методы ассимиляции данных Д3З в модели развития пожара. Ассимиляции данных предполагает совместное использование информации фактических наблюдений и модельных оценок с учетом их погрешностей для определения наиболее вероятной реальной динамики процесса. Применительно к природным пожарам данный подход позволяет уточнить оценку положения фронта горения, настроить значения параметров модели и улучшить прогноз дальнейшей динамики распространения огня [33][34].

Модели распространения огня также применяются для моделирования глобального изменения частоты и интенсивности пожаров в моделях земной системы. Комбинирование упрощенных моделей распространения огня и моделей возникновения и ликвидации пожаров позволяет оценить их влияния на наземные экосистемы и содержание парниковых газов в атмосфере в контексте глобального изменения климата [35]. Спутниковые данные позволяют формировать многолетние архивы площадей пожаров и частоты их возникновения [36], которые используются для оценки адекватности глобальных моделей природных пожаров и их настройки [35].

Ряд экспериментов по моделированию развития природных пожаров с использоваием результатов обработки данных ДЗ3 выполнен авторами данной статьи на основе инфраструктуры Центра коллективного пользования (ЦКП) «ИКИ-Мониторинг» [37] при финансовой поддержке 
государства в лице Минобрнауки России: соглашение 05.577.21.0294 «Разработка технологий автоматизированной обработки спутниковых данных дистанционного зондирования Земли для создания и поддержки информационных сервисов мониторинга лесных ресурсов и охотничьих угодий России», уникальный идентификатор проекта RFMEFI57718X0294.

\section{References}

[1] Grishin A.M. Mathematical models of forest fires, 1981. (In Russian).

[2] Grishin A.M., Penenko V.V. Mathematical modelling of forest fires and new methods to fight them, Nauka, 1992. (In Russian).

[3] Sullivan A., A review of wildland fire spread modelling, 1990-present, 1: Physical and quasi-physical models, International Journal of Wildland Fire, 2009, 18(4) pp. 349-368. DOI: 10.1071/WF06143.

[4] Potter B. Atmospheric interactions with wildland fire behaviour - II. Plume and vortex dynamics, International Journal of Wildland Fire, 2012, 21(7), pp. 802-817. DOI: 10.1071/WF11129.

[5] Potter B. Atmospheric interactions with wildland fire behaviour - I. Basic surface interactions, vertical profiles and synoptic structures, International Journal of Wildland Fire, 2012, 21(7), pp. 779-801. DOI: 10.1071/WF11128.

[6] Dorrer G.A. Dynamic of forest fires, Izdatel'stvo sibirskogo otdeleniya rossijskoj akademii nauk 2008. (In Russian).

[7] Rothermel R. A mathematical model for predicting fire spread in wildland fuels, p. 48.

[8] Rothermel R. Predicting behavior and size of crown fires in the northern Rocky Mountains, Ogden, UT: U.S. Department of Agriculture, Forest Service, Intermountain Research Station, 1991, INT-RP-438. DOI: 10.2737/INT-RP-438.

[9] Sullivan A. A review of wildland fire spread modelling, 1990-present 3: Mathematical analogues and simulation models, International Journal of Wildland Fire, 2009, 18(4), pp. 387-403 DOI: 10.1071/WF06144.

[10] Cruz M., Alexander M., Sullivan A., Gould J., Kilinc M. Assessing improvements in models used to operationally predict wildland fire rate of spread, Environmental Modelling \& Software, 2018, 105, pp. 54-63 DOI: 10.1016/j.envsoft.2018.03.027.

[11] Sullivan A. A review of wildland fire spread modelling, 1990-present 2: Empirical and quasi-empirical models, International Journal of Wildland Fire, 2009, 18(4), pp. 369-386 DOI: 10.1071/WF06142.

[12] Tymstra C., Bryce R.W., Wotton B.M., Taylor S.W., Armitage O.B. Development and structure of Prometheus the Canadian Wildland Fire Growth Simulation Model, Edmonton: Northern Forestry Centre, 2010, OCLC: 813589652.

[13] Finney M., Grenfell I., McHugh C., Seli R., Trethewey D., Stratton R., et al. A Method for Ensemble Wildland Fire Simulation, Environmental Modeling \& Assessment, 2011, 16(2), pp. 153-167. DOI: 10.1007/s10666-010-9241-3.

[14] Khvostikov S.A., Bartalev S.A., Loupian E.A. Stochastic wildfire model based on Monte-Carlo method and remote sensing data integration, Sovremennye problemy distantsionnogo zondirovaniya Zemli iz kosmosa, 2016, 13(5), pp. 145-156. DOI: 10.21046/2070-7401-2016-13-5-145-156. (In Russian).

[15] Andrews P. Do you BEHAVE? Application of the BehavePlus fire modeling system, 2010, p. 17.

[16] Finney M. FARSITE: Fire Area Simulator-model development and evaluation, Ft. Collins, CO: U.S. Department of Agriculture, Forest Service, Rocky Mountain Research Station, 1998, RMRS-RP-4. DOI: 10.2737/RMRS-RP-4.

[17] Jarvis A., Reuter H., Nelson A., Guevara E. Hole-filled SRTM for the globe Version 4, 2008. 
[18] Tachikawa T., Hato M., Kaku M., Iwasaki A. Characteristics of ASTER GDEM version 2, In 2011 IEEE International Geoscience and Remote Sensing Symposium. Vancouver, BC, Canada: IEEE, 2011, pp. 3657-3660. DOI: 10.1109/IGARSS.2011.6050017.

[19] Arroyo L., Pascual C., Manzanera J.. Fire models and methods to map fuel types: The role of remote sensing, Forest Ecology and Management, 2008, 256(6), pp. 1239-1252. DOI: 10.1016/j.foreco.2008.06.048.

[20] Reeves M., Ryan K., Rollins M., Thompson T. Spatial fuel data products of the LANDFIRE Project, International Journal of Wildland Fire, 2009, 18(3), pp. 250. DOI: 10.1071/WF08086.

[21] Giglio L., Schroeder W., Justice C. The collection 6 MODIS active fire detection algorithm and fire products, Remote Sensing of Environment, 2016, 178, pp. 31-41. DOI: 10.1016/j.rse.2016.02.054.

[22] Csiszar I., Schroeder W., Giglio L., Ellicott E., Vadrevu K., Justice C., et al. Active fires from the Suomi NPP Visible Infrared Imaging Radiometer Suite: Product status and first evaluation results, Journal of Geophysical Research: Atmospheres, 2014, 119(2), pp. 803-816. DOI: 10.1002/2013JD020453.

[23] Oliva P., Schroeder W. Assessment of VIIRS 375m active fire detection product for direct burned area mapping, Remote Sensing of Environment, 2015, 160, pp. 144-155. DOI: 10.1016/j.rse.2015.01.010.

[24] Anderson K., Englefield P., Little J., Reuter G. An approach to operational forest fire growth predictions for Canada, International Journal of Wildland Fire, 2009, 18(8), pp. 893. DOI: 10.1071/WF08046.

[25] Khvostikov S.A., Balashov I.V., Bartalev S.A., Efremov E.Yu., Loupiyan E.A. Regional scale optimization of wildfire model parameters and modeling of wildfire dynamic using remote sensing data, Sovremennye Problemy Distantsionnogo Zondirovaniya Zemli iz Kosmosa, 2012, 9(3), pp. 91-100. (In Russian).

[26] Bartalev S.A., Egorov V.A., Efremov V.Yu., Loupian E.A., Stytsenko F.V., Flitman E.V. Integrated burnt area assessment based on combine use of multi-resolution MODIS and Landsat-TM/ETM+ satellite data, Sovremennye Problemy Distantsionnogo Zondirovaniya Zemli iz Kosmosa, 2012, 9(2), pp. 9-26. (In Russian).

[27] Filippi J., Mallet V., Nader B. Evaluation of forest fire models on a large observation database, Natural Hazards and Earth System Science, 2014, 14(11), pp. 3077-3091. DOI:10.5194/nhess-14-3077-2014.

[28] Sa A., Benali A., Fernandes P., Pinto R., Trigo R., Salis M. Evaluating fire growth simulations using satellite active fire data. Remote Sensing of Environment. 2017, 190, pp. 302-317. DOI: 10.1016/j.rse.2016.12.023.

[29] Lautenberger C. Wildland fire modeling with an Eulerian level set method and automated calibration, Fire Safety Journal, 2013, 62, pp. 289-298. DOI: 10.1016/j.firesaf.2013.08.014.

[30] Noonan-Wright E., Opperman T., Finney M., Zimmerman G., Seli R., Elenz L. Developing the US Wildland Fire Decision Support System, Journal of Combustion, 2011, 2011, pp. 1-14. DOI: $10.1155 / 2011 / 168473$.

[31] Mavsar R., Gonzalez Caban A., Varela E. The state of development of fire management decision support systems in America and Europe, Forest Policy and Economics, 2013, 29, pp. 45-55. DOI: 10.1016/j.forpol.2012.11.009.

[32] Bartalev S.A., Stytsenko F.V., Khvostikov S.A., Loupian E.A. Methodology of post-fire tree mortality monitoring and prediction using remote sensing data, Sovremennye problemy distantsionnogo zondirovaniya Zemli iz kosmosa, 2017, 14(6), pp. 176-193. DOI: 10.21046/2070-7401-2017-14-6-176-193 (In Russian)

[33] Rochoux M., Emery C., Ricci S., Cuenot B., Trouve A. Towards predictive data-driven simulations of wildfire spread \&ndash; Part 2: Ensemble Kalman Filter for the state estimation of a front-tracking simulator of wildfire spread, Natural Hazards and Earth System Sciences Discussions, 2014, 2(5), pp. 3769-3820. DOI: 10.5194/nhessd-2-3769-2014.

[34] Mandel J., Bennethum L., Beezley J., Coen J., Douglas C., Kim M., et al. A wildland fire model with data assimilation, Mathematics and Computers in Simulation, 2008, 79(3), pp. 584-606. DOI: 10.1016/j.matcom.2008.03.015

[35] Hantson S., Arneth A., Harrison S., Kelley D., Prentice I., Rabin S., et al. The status and challenge of global fire modelling, Biogeosciences Discussions, 2016, pp. 1-30. DOI: 10.5194/bg-2016-17.

[36] Giglio L., Randerson J., van der Werf G., Kasibhatla P., Collatz G., Morton D., et al. Assessing variability and long-term trends in burned area by merging multiple satellite fire products, Biogeosciences, 2010, 7(3), pp. 1171-1186. DOI: 10.5194/bg-7-1171-2010. 
[37] Loupian E.A., Proshin A.A., Burtsev M.A., Balashov I.V., Bartalev S.A., Efremov V.Yu., Kashnitskiy A.V., Mazurov A.A., Matveev A.M., Sudneva O.A., Sychugov I.G., Tolpin V.A., Uvarov I.A. IKI center for collective use of satellite data archiving, processing and analysis systems aimed at solving the problems of environmental study and monitoring, Sovremennye problemy distantsionnogo zondirovaniya Zemli iz kosmosa, 2015, 12(5), pp. 263-284. (In Russian). 